\title{
2 \\ The Mind As A Reliable Switch: Challenges Of Rapidly Controlling Devices Without Prior Learning
}

\author{
Ashley Craig, ${ }^{*}$ Les Kirkup ** Paul McIsaac * and Andrew Searle ** \\ *Department of Health Sciences, ** Department of Applied Physics \\ Faculty of Science, University of Technology, Sydney \\ a.craig@uts.edu.au; kirkup@phys.uts.edu.au; asearle@phys.uts.edu.au
}

\begin{abstract}
Activity within the brain causes electrical potentials to exist on the scalp. The study of these potentials is generally referred to as electroencephalography (EEG). EEG is widely used in medical diagnosis and in biofeedback studies in which a person can learn to control some element of their EEG spectrum, usually in response to some visual stimulus. This paper reports the control of the alpha component of the EEG spectrum which does not require the learning that biofeedback demands. We show that participants can achieve rapid and reliable remote control of electrical devices using increase in alpha wave activity associated with reduced visual input. The physiological basis, technical achievements and challenges, and applications will be discussed.
\end{abstract}

KEYWORDS Alpha wave, switch, electroencephalograph, environmental control, noise suppression

\section{INTRODUCTION}

Controlling the environment with our mind has always been a wish of humankind. Early attempts to achieve this using thoughts or 'willpower' alone has been called telekinesis. Though telekinesis is being investigated scientifically (McCrone, 1994), the raw electrical signal in the brain is just too small to have any direct influence over objects external to the brain. Nonetheless, 'mind control' remains a possibility for controlling electrical devices because neural activity is associated with signals called electroencephalograph waves (EEG; Fisch, 1991). 'Biofeedback' has been used to control electrical devices by providing visual or auditory feedback of the EEG signal to the person. The goal of EEG biofeedback would be to enhance the conscious manipulation of the EEG spectrum, such as increasing or decreasing alpha levels (which is in the frequency range of 8 to $12 \mathrm{~Hz}$ ). For instance, using EEG signal feedback, people have been able to control a cursor on a computer screen, transmit Morse code messages, start a robot or model electric train by raising their alpha levels, or stop it by decreasing levels (Bozinovski, Sestakov \& Bozinovska, 1988). People have also been able to communicate by varying their high and low EEG frequencies (Elder, Lashley, Kedouri, Regenbogen, Martyn, Roundtree, \& Grenier, 1986). Unfortunately, biofeedback has slow response times and usually takes at least 8-12 hours to learn control over their physiological responses (Craig \& Cleary, 1982). In the experience of the authors, few people have the ability to consistently and quickly control their EEG (alpha) levels, especially when stressed. These problems limit the usefulness of biofeedback in reliably controlling devices like a computer or lamp.

An alternative strategy for 'mind control' involves using increases in alpha EEG wave activity amplitude levels (synchronisation) associated with a reduction in visual input (eyes closed), and alpha level decreases

Human-Computer Interaction: INTERACT'97 S. Howard, J. Hammond \& G. Lindgaard (editors)

Published by Chapman \& Hall OIFIP 1997 
(desynchronisation) associated with an increase in visual input (eyes opened). This paper reports work that builds upon the research of Dewan (1967) who published 30 years ago on environmental control and the conscious control of alpha levels using this strategy. Scientists in the Faculty of Science of the University of Technology, Sydney have recently made an advance in this area following detailed investigation of the response of 31 subjects' EEG to various stimuli. They observed that all subjects were producing reproducible and consistent EEG signal responses in the alpha range $(8$ to $11.5 \mathrm{~Hz}$ ) linked with closure of eyes. This was unexpected as there are few reports of EEG being predicted or manipulated consistently and reliably. This led the scientists to believe that intentional and reliable control over the environment is a possibility. As an consequence of this research, technology has been developed which allows electrical devices or appliances to be immediately and remotely operated by a person through the conscious control of specific signals generated by the brain. With further research and development, a person will have a sophisticated environmental control device. It was labelled "the ultimate interface between man and machine" (Australian Financial Review, 1996).
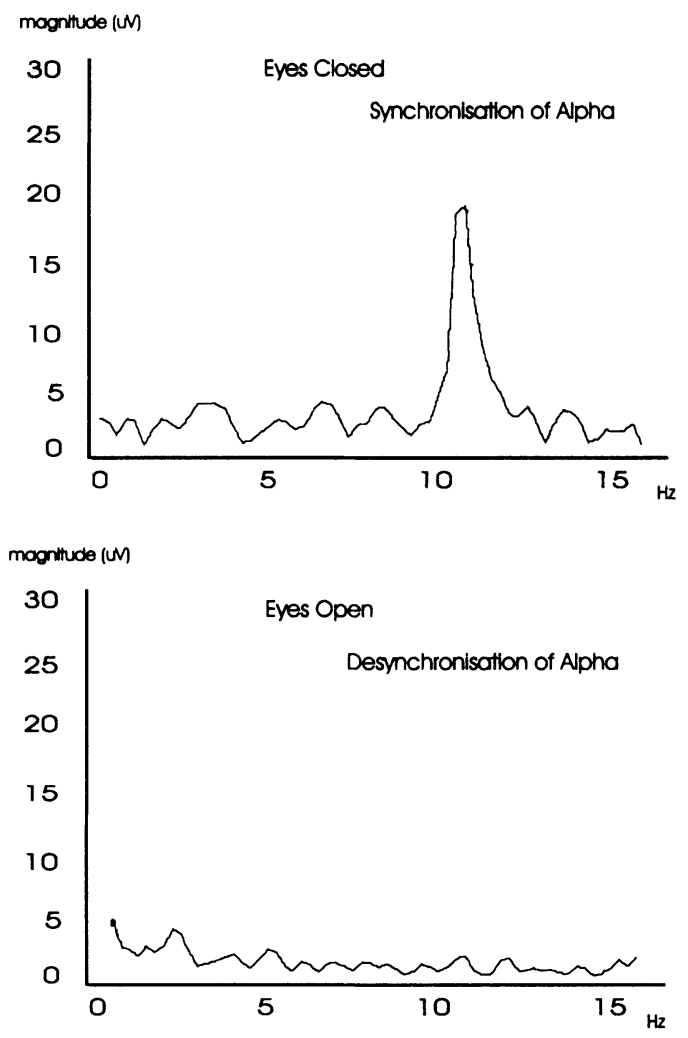

Figure 1 Showing spectral analyses of right hemisphere EEG up to $16 \mathrm{~Hz}$ from the visual cortex. Synchronisation and desynchronisation of alpha associated with reduced visual input (eyes closed) and increased visual input (eyes open) respectively is shown at $10.5 \mathrm{~Hz}$ for a subject assessed in our laboratories. The difference in alpha amplitude at $10.5 \mathrm{~Hz}$ between eyes closed and eyes open is the basis for the conscious control of devices. 
The aim of this paper is to present the physiological background for the nature of the EEG phenomenon harnessed by the technology, to discuss the technical achievements and challenges, and to conclude with a brief description of possible applications.

\section{PHYSIOLOGICAL BACKGROUND OF}

\section{ALPHA EEG ACTIVITY}

The physiological importance of the alpha EEG wave remains unknown (Shaw, 1992). While the alpha wave occurs all over the cortex, it is believed to be prominent in the posterior (visual or occipital cortex) and central/ parietal regions. It is also known that attentiveness, concentration, anxiety and visual fixation reduce the amplitude of alpha levels (called desynchronisation) (Mulholland, 1995). Alpha levels are increased (or synchronised) when one reduces visual input by closing the eyes, rolls the eyes upwards or when one relaxes (Craig, McIsaac, Kirkup, Searle \& Moses, in press; Mulholland \& Evans, 1966). It is

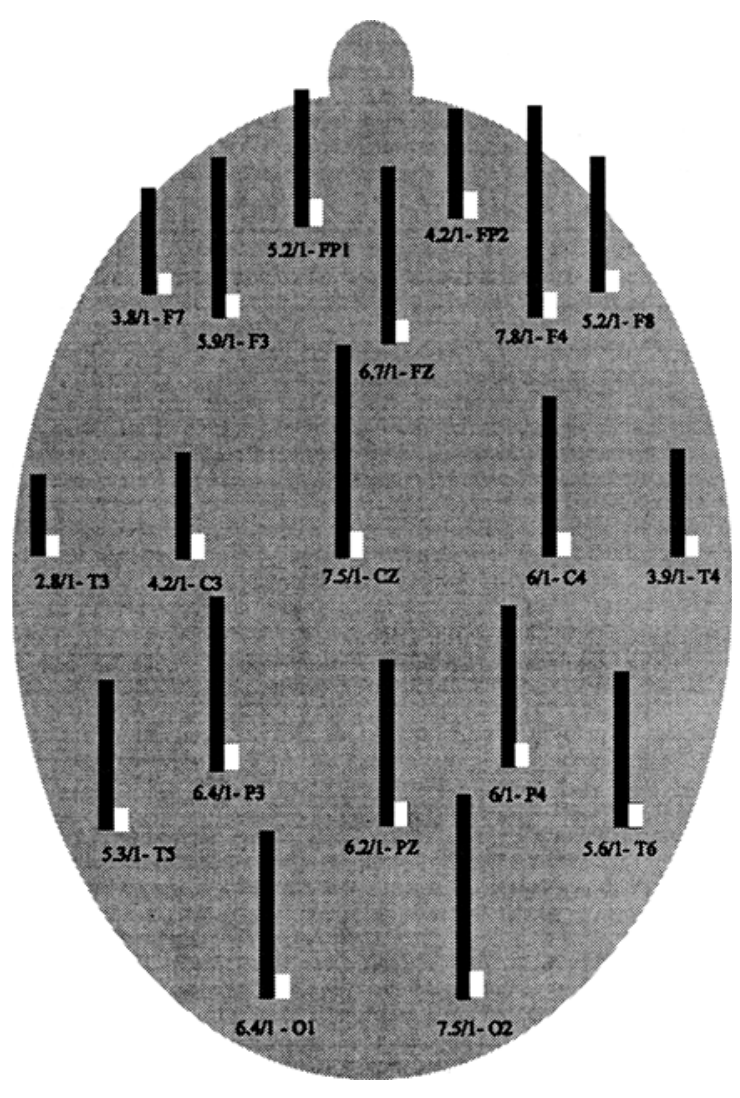

EYES CLOSED EYES OPEN

Figure 2 shows the ratio of alpha increases contingent with eye closure with alpha levels when eyes are open, for all the cortex (average responses for 21 participants). 
possible that the alpha level increases seen during reduced visual input is a function of decreased activity in oculomotor control systems in the brain. With reduced visual input, scanning and visual attention decrease. Alpha wave amplitude reduction is associated with opening the eyes, increasing visual scanning activity and attentiveness and this is most likely the result of increased cortical oculomotor activity. Alpha levels decrease when cortical activation increases. In central cortical sites, alpha wave levels may also be decreased during high levels of mental attention and non-occipital alpha rhythm, called mu alpha (seen in central areas), is also decreased by cortical activation associated with the muscle movement (Mulholland, 1995).

The synchronisation of the alpha rhythm has been studied extensively in our laboratories in a number of male and female subjects of varied ages from children to older age persons (Craig et al., in press). A typical alpha wave synchronisation response is illustrated in
Figure 1 which shows the alpha wave magnitude when eyes are closed for one participant. This response was recorded from the occipital cortex (right hemisphere) at $10.5 \mathrm{~Hz}$. The sharp increase in alpha wave magnitude contingent with eye closure seen in this person generally occurs all over the cortex, and this increase, (calculated from "eyes open" alpha levels), is usually of the order of a 300 to $1000 \%$ increase. Substantial increases in alpha resulted contingent with eye closure and this occurred all over the cortex. Alpha wave level increases associated with eye closure were shown to be greatest in occipital $\left(\mathrm{O}_{1}, \mathrm{O}_{2}\right)$, central $\left(\mathrm{C}_{3}, \mathrm{C}_{4}, \mathrm{C}_{\mathrm{z}}\right)$, parietal $\left(\mathrm{P}_{\mathrm{z}}, \mathrm{P}_{3}, \mathrm{P}_{4}\right)$, and frontal areas $\left(\mathrm{F}_{\mathrm{z}}, \mathrm{F}_{3}, \mathrm{~F}_{4}\right)$. Figure 2 further illustrates alpha level increases all over the cortex in contrast to alpha levels when eyes are open. The majority of subjects assessed in our laboratory show alpha synchronisation contingent with eye closure in the 9.5 to 11 Hertz range while a minority show eye closure alpha synchronisation in the 8.5 to $9.5 \mathrm{Hertz}$ region.

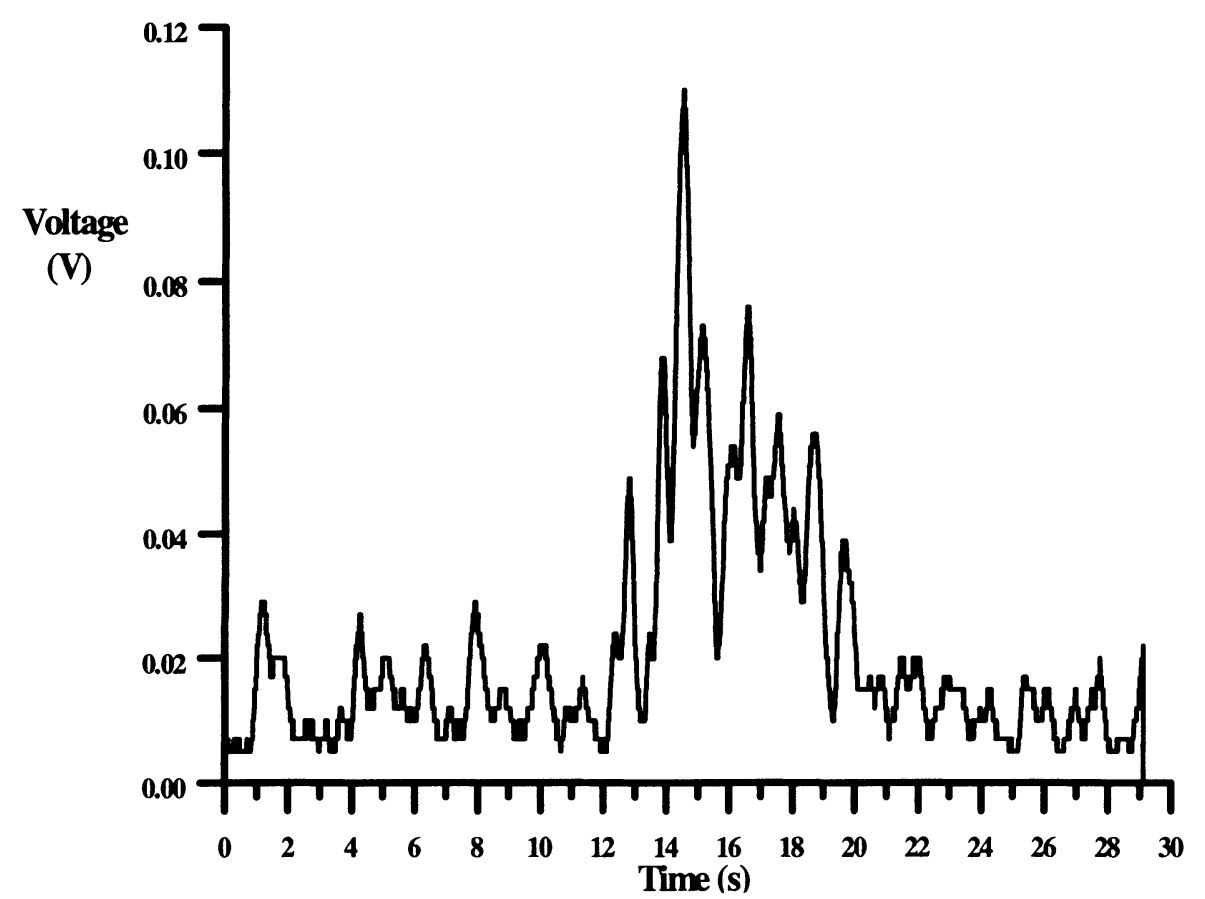

Figure 3 Variation in the amplitude of the $10 \mathrm{~Hz}$ component of the EEG spectrum. The subject closed his eyes at $\mathbf{t}=\mathbf{1 2} \mathrm{s}$ 
There were no differences between the right and left hemispheres in synchronisation of alpha, nor were there any significant differences in the size of the alpha increases associated with eye closure in any of the cortical sites between males and females, or age within the age range for the subjects. Handedness was also not significantly related to alpha eye closure responses.

\section{TECHNOLOGY DEVELOPMENT}

For many individuals, the ratio of the (average) amplitude of the alpha component of the EEG spectrum between eye closed to eyes open is about 2 to $3 \mathrm{~s}$ (Craig et al. in press). Such a significant increase in this component upon eye closure facilitates the development of a system designed to detect and utilise the change.

Essential elements of the system are signal amplification, filtering and smoothing. The 'raw' EEG signal at $\approx 10 \mathrm{~Hz}$ is typically in the range 10 to $50 \mu \mathrm{V}$. Therefore in order to allow other operations to be reliably performed on the signal, such as comparison with a d.c reference, it is convenient to apply a gain to the signal of around $100 \mathrm{~dB}$. A bandpass filter with centre frequency at $10 \mathrm{~Hz}$ and bandwidth of $2 \mathrm{~Hz}$ adequately spans the frequencies at which most subjects show a significant increase between eyes open and eyes closed.

Figure 3 shows the variation in the amplitude of the EEG signal over a period of $30 \mathrm{~s}$. At $\mathrm{t}=12 \mathrm{~s}$ the subject closed his eyes. At this point in time a marked increase in the signal amplitude can clearly be seen. However, due to this signal's large variation, any switching system based on this amplitude variation is likely to suffer from intermittent switching. In order to largely eliminate spurious switching, a simple averager with a time constant of about $2 \mathrm{~s}$ is applied to the amplified and filtered. signal.

Figure 4 shows the smoothed output of the signal averager. The smoothed signal can now be used as an input to a signal comparator which switches the state of the device (such as a lamp) once the EEG signal has exceeded some predetermined value. Further details of the system can be found elsewhere (Kirkup, Searle, Craig, McIsaac and Moses, in press). An attractive feature of the system is that it has a single adjustable parameter: the threshold voltage at which switching

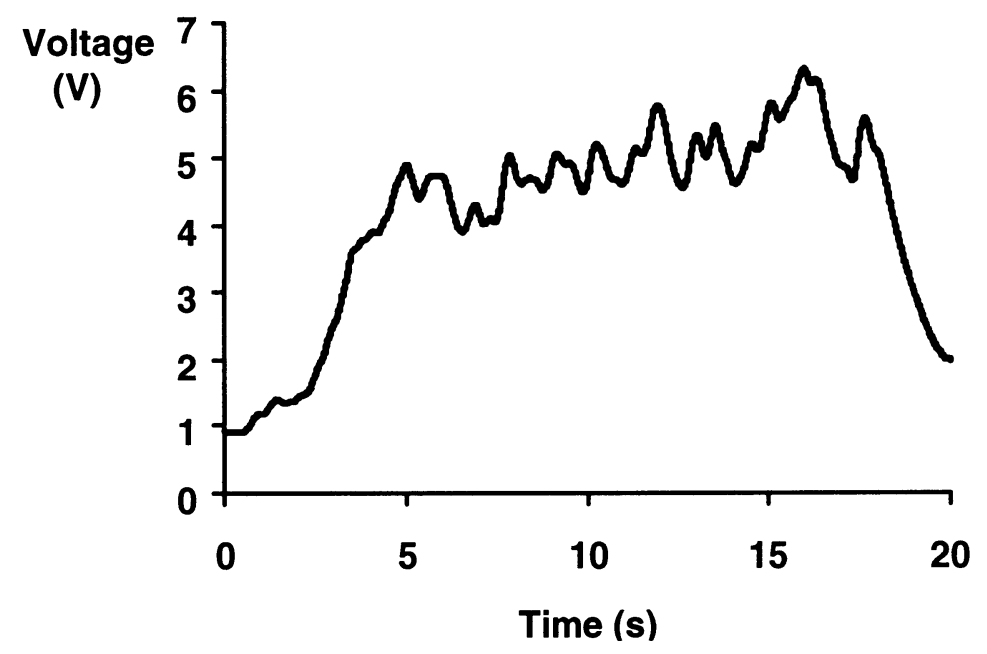

Figure 4 The smoothed output of the system. The subject closed his eyes at $t=2 \mathrm{~s}$. Between approximately $3 \mathrm{~s}$ and $5 \mathrm{~s}$ the voltage increases approximately proportionally with time. 
occurs. It has been noted in a longitudinal study of one subject over 24 months that, once set, the threshold setting requires no further adjustment. The average time for switching after eye closure is around 2 to $3 \mathrm{~s}$ for the subjects tested. Figure 4 shows that the voltage increase after eye closure is approximately proportional with time, suggesting that proportional control or multilevel switching is possible. Indeed the basic system has been developed to the stage where two level switching has been demonstrated. By setting two switching levels, an alpha signal can control a toy slot car. The car may be started, stopped and its speed toggled high and low through control of the amplitude of the smoothed EEG signal. The time delay between eye closure and detection of the increase in the alpha signal is a matter of great importance as it affects the number of switching operations that may be performed in a given time. It is not known at this time whether repeated attempts to switch devices on and off results in a decrease in the mean switching time, though early indications do suggest a marginal decrease in the mean time with habituation. We are currently evaluating a number of other methods of detecting the change in the alpha signal level which may yield mean switching time between 1 and $2 \mathrm{~s}$. Our studies show that the occurrence of the alpha change can differ significantly from person to person. Specifically, upon eye closure, some subject show 'bursts' of alpha which are sustained so long as the eyes remain closed, while others burst momentarily upon eye closure only to relax to the 'eyes open' level before bursting again. It seems likely that different methods of alpha detection will suit those who exhibit continuous or intermittent alpha enhancement.

\section{TECHNOLOGICAL CHALLENGES}

A major issue to be addressed is that of spurious switching which is not related to the natural variation in the alpha signal that exists with eyes open or closed, but is due to the large excursions in signal amplitude caused by other sources of electrical activity at $10 \mathrm{~Hz}$. An example of such a source is due to head movement (e.g. nodding or rotation) which generates electrical signals due to muscle activity (often referred to as EMG). EMG generally has a wide frequency spectrum ranging from close to d.c. to beyond $100 \mathrm{~Hz}$. Though much of the energy in the EMG spectrum lies beyond $15 \mathrm{~Hz}$ (Sadasivan and Dutt, 1995), enough normally exists at $10 \mathrm{~Hz}$ to swamp the true EEG signal. If a system, for example, is to be built that will be used to assist severely physically disabled, it is of paramount importance to address the issue of electrical noise. We have devised a simple method which relies on the fact that EMG signals have a recognisable electrical 'signature' (Kirkup et al. 1997). When the EMG sources are active this signature is detected and the output of the system is 'frozen' so that no spurious switching can occur. The system becomes 'live' again once the noise component has relaxed below some predetermined threshold. This approach has the attraction of simplicity and real time signal processing, but the disadvantage that the system is insensitive to real changes in EEG signal while the system is frozen. We are currently developing other methods of reducing the effect of noise signals which does not cause the output of the system to be disabled.

Perhaps more challenging than the suppression of noise is the issue of electrode design. Conventional electrodes are generally constructed from silver/silver chloride and require the use of conducting paste or gel in order to assure that good, low impedance electrical contact is established and maintained between electrode and scalp. In many clinical applications of EEG the scalp must be prepared by scraping with a blunt needle or similar in order that the impedance is $2 \mathrm{k} \Omega$ or less (Fisch, 1991). Though the requirements of our system are less exacting (impedance of less than $20 \mathrm{k} \Omega$ is quite adequate), the challenge of providing good electrical contact remains. 'Wet' electrodes using conducting gel dry out over time and in doing so tend to impair the quality of the electrical contact. Additionally, wet electrodes are not viewed favourably by users. We are pursuing the possibility of creating dry electrodes in which EEG signals are coupled capacitively between scalp and electrode (Taheri, Knight and Smith, 1994). These offer the advantages of no skin preparation nor the use of conducting gel, but introduce their own problems such as very high electrode impedances which are susceptible to electromagnetic interference. We believe that significant improvements in electrode design are demanded if this technology is to enjoy widespread application.

\section{APPLICATIONS}

There are many possible applications of environmental control using brain signals. For instance, disabled people who have limited control of devices with their 
arms and hands, would benefit greatly if they could remotely and quickly control a range of devices using their brain. Switches already exist that allow some control, for example, eye blinking and infra-red signals and breath activated switches. Voice activated systems especially offer a promising environmental control system. However, this strategy would not be useful for persons with voice disorders. There are significant problems when the voice is altered due to say the common cold. Additionally, the technology requires extensive training and it could not be used easily in a noisy environment. The issue is one of providing a range of switches that can be utilised by people who are disabled. Controlling the environment with alpha synchronisation provides another potentially versatile strategy for enhancing environmental control for those with disabilities such as spinal chord injury and cerebral palsy. The alpha wave switch would obviously require the person using the switch, to have a significant alpha synchronisation and to be able to close their eyes on demand.

Possible further applications are:

(a) basis of an environmental control system in the house of the future, or possibly for environmental control in space.

(b) hands free industrial applications.

(c) computing and multimedia purposes,

(d) communication purposes,

(e) recreational pastimes, such as interactive computer games

\section{ACKNOWLEDGMENT}

The authors would like to acknowledge the part funding of this work by the Motor Accident Authority of New South Wales, Australia

\section{REFERENCES}

Bozinovski, S., Sestakov, M., \& Bozinovska, L. (1988). Using EEG alpha rhythm to control a mobile robot. IEEE Engineering in Medicine \& Biology Society, 10th Annual International Conference, 1515 CH2566.

Craig, A. R., \& Cleary, P. J. (1982). Reduction of stuttering by young male stutterers using EMG feedback. Biofeedback and Self-Regulation 7, 241255.

Craig, A., McIsaac, P., Kirkup, L., Searle, A., \& Moses, P. (in press). Brain signals and control of electrical devices. Today's Life Science.

Dewan, E. M. (1967). Occipital alpha rhythm eye position and lens accommodation. Nature, 214, 975977.

Elder, S. T., Lashley, J. K., Kedouri, N., Regenbogen, D., Martyn, S., Roundtree, G. \& Grenier, C. (1986). Can subjects be trained to communicate through the use of EEG biofeedback? Clinical Biofeedback and Health, 9, 42-47.

Fisch, B. (1991). Spehlmann's EEG primer (2nd Ed). Amsterdam: Elsevier.

Kirkup, L., Searle, A., Craig, A., McIsaac, P. \& Moses, P. (in press). EEG based system for rapid on-off switching without prior learning. Medical and Biological Engineering and Computing.

McCrone, J. (1994) Psychic powers. What are the odds? New Scientist, November 34-38.

Mulholland, T. (1995). Human EEG, behavioral stillness and biofeedback. International Journal of Psychophysiology, 19, 263-279.

Mulholland, T., \& Evans, C. R. (1966). Oculomotor function and the alpha activation cycle. Nature, 211, 1278-1279.

Sadasivan, P.K. and Dutt, D.N. (1995): Use of finite wordlength FIR digital filter structures with improved magnitude and phase characteristics for reduction of muscle noise in EEG signals. Medical and Biological Engineering and Computing. 33 pp. 306-312

Shaw, J. C. (1992). The ubiquitous alpha rhythm- a selective review. Journal of Electrophysiology Technology, 18, 5-27.

Taheri, B., Knight, R. and Smith, R. (1994) A Dry Electrode for EEG recording. Electroencephalography and Clinical Neurophysiology, 90, pp376-383. 